\title{
LOS TRATADOS FILOSÓFICOS DE S. VICENTE FERRER: NOTA HISTÓRICA Y BIBLIOGRÁFICA
}

\author{
José Ángel Garcia-Cuadrado \\ Universidad de Navarra
}

El pensamiento medieval europeo se encuentra a finales del siglo XIV en un momento especialmente delicado, en donde los modelos metafísicos escolásticos han comenzado a entrar en crisis por la introducción de un nuevo modo de pensar que tiene como punto de referencia la lógica nominalista. Es el momento, además, en donde empieza a despuntar el interés humanista característico del Renacimiento, alejado de la especulación metafísica que había comenzado a degenerar en un ejercicio dialéctico tan sutil como vacío. El pensamiento hispano no se mantiene al margen de esta situación conflictiva, y en el seno de las universidades apenas recién creadas, se dan cita maestros de escuelas realistas - tanto dominicos tomistas como franciscanos escotistas- y nominalistas, junto con autores lulistas, con concepciones a menudo radicalmente diversas acerca de los temas fundamentales de la especulación filosófica.

Dentro de este complejo mosaico se encuadran los tratados filosóficos de S. Vicente Ferrer que han suscitado el interés de historiadores del pensamiento medieval en general, y de la historia de la lógica en particular. En los últimos años se ha comenzado a prestar una mayor atención a estas obras de Ferrer: en 1977 se publica la primera edición crítica del Tractatus de Suppositionibus. A esta edición hay que sumar la traducción castellana de las dos obras lógicas que se conservan de Ferrer, así como numerosos artículos y estudios centrados en algunos aspectos parciales de la doctrina contenida en dichos tratados. Pero antes de seguir adelante, es necesario presentar a nuestro autor y su obra filosófica dentro del contexto histórico e intelectual de la época.

\section{CONTEXTO HISTÓRICO-FILOSÓFICO DEL SIGLO XIV}

Después del fecundo siglo XIII, la Universidad de París, comienza a vivir una profunda crisis en su estructura. La presión de los monarcas franceses en favor de una progresiva 
nacionalización de la Universidad, corre paralela a la sustracción de la influencia de los papas. ${ }^{1}$ Por otro lado, las divergencias se acentúan entre los filósofos y teólogos de las distintas órdenes. Ya en el año 1309, el Capítulo General de los Dominicos, reunido en Zaragoza, adopta a Santo Tomás como doctor oficial de la orden; los agustinos, por su parte, habían elegido a Egidio Romano como teólogo oficial desde 1287. Los fransciscanos se encontraban de hecho divididos entre los seguidores de San Buenaventura y los de Duns Escoto. ${ }^{2}$

Las causas de este confuso panorama son variadas y complejas: las condenaciones de 1277, las disputas parisinas entre los diversos maestros, las interpretaciones árabes del pensamiento aristotélico, etc... Quizás, como apunta Gilson, el problema de la armonización entre fe y razón no había sido asumido por igual en todos los pensadores de las distintas órdenes. ${ }^{3}$ Para algunos de ellos, la introducción de la filosofía aristotélica en el pensamiento cristiano suponía una concesión a la filosofía pagana. Lo que sí parece cierto es que en el ambiente intelectual de las universidades europeas comienza a respirarse un aire de cierta desconfianza en la razón junto con una implícita tendencia fideísta. ${ }^{4}$

En este ambiente de desconfianza en la razón surge un nuevo estilo de pensar, extremadamente crítico de la filosofía del XIII, que dará como resultado la contraposición entre los antiqui y los moderni, o realistas y nominalistas. Entre los seguidores de la via moderna, Ockham ocupa un puesto principal. Para comprender el alcance de esta nueva actitud intelectual es preciso acudir a la discusión teológica. En efecto, según Ockham y los moderni, la existencia de verdades necesarias y eternas (tal como se entiende entre los filósofos aristotélicos del XIII, muchos de los cuales acusan un fuerte influjo árabe), limita de algún modo la libertad y omnipotencia divina. Por eso, estos autores «modernos» se decantan hacia una actitud marcadamente voluntarista frente al «intelectualismo» propugnado por los filósofos del siglo anterior. ${ }^{5}$

Las reacciones contra la doctrina «moderna» no se hicieron esperar, y en 1339 se prohíbe con energía la enseñanza de los textos de Ockham en la Universidad de París. Ya con anterioridad, en 1324, el Venerabilis Inceptor, había sido llamado a Avignon por el papa Juan XXII para responder a una querella de enseñanza herética. El proceso teológico contra Ockham no llegó a concluirse; pero es interesante constatar que así como la doctrina teológica fue rápidamente contestada, los presupuestos filosóficos que en ella subyacen se conservaron intactos. ${ }^{6}$

1 Cfr. Gilson, E., La filosofía en la Edad Media, Gredos, Madrid, 1980, pp. 656-659.

2 Ibidem.; para una información histórica del momento que nos ocupa cfr. Rábade, S., Guillermo de Ockham y la filosofía del siglo XIV, CSIC, Madrid 1966; Torrelló, R.M., «El ockamismo y la decadencia escolástica en el siglo XIV», (I) en Pensamiento, 9 (1953), pp. 199-228; (II) en Pensamiento, 11 (1955), pp. 171-188; (III) en Pensamiento, 11 (1955), pp. 259-283.

3 Cfr. Gilson, E., La filosofía en la Edad Media, pp. 591-592.

4 Fraile, G., Historia de la Filosofía (II-2.9), BAC, Madrid 1966, p. 1051.

5 Cfr. Gilson, E., La filosofía en la Edad Media, pp. 603-607.

6 Cfr. Torrelló, R.M., «El ockhamismo y la decadencia... (I)», p. 202. 
Y es precisamente en sus raíces filosóficas donde se encuentra el gran corte realizado por Ockham y los nominalistas respecto a la tradición anterior, aunque ya en la filosofía de Duns Escoto se encuentran presentes todos los presupuestos que Ockham llevó hasta sus últimas consecuencias. En los años precedentes se había asistido a las disputas entre dominicos aristotélicos y franciscanos agustinianos pero con unos principios realistas comunes. Ahora el nominalismo niega el valor real del universal y concede la prioridad absoluta al individuo, como único soporte del saber real. De este modo se encuentran dos posturas filosóficas y teológicas enfrentadas: los realistas y los nominalistas. ${ }^{7}$ La cuestión de los universales había sido objeto de encendidas discusiones en los siglos precedentes; pero es en este momento histórico donde se definen las distintas posturas con perfiles netos, y los contrastes entre las diversas escuelas se presentan ahora con tonos enérgicos y polémicos. Los moderni sostienen que el valor absoluto de realidad se contiene en el individuo concreto y particular; el universal no será para ellos más que una entidad mental que utilizamos de modo arbitrario para designar al conjunto de los individuos existentes. En reacción a los nominalistas, surgieron las posturas realistas exageradas, que conceden una existencia extramental a los conceptos universales. Entre los nominalistas y los realistas exagerados, se encuentran los realistas moderados, que sostienen - continuando la línea aristotélica de Tomás de Aquino-que el universal posee una existencia mental, pero con un fundamento en la realidad. El universal no existe independientemente de los particulares, sino que se halla multiplicado en todos ellos. Es en este contexto intelectual donde se sitúa la actividad de Vicente Ferrer como filósofo. Dentro del realismo tomista, desarrollará su etapa docente en la Universidad de Lérida, punto de encuentro de las teorías nominalistas, realistas y lulistas.

\section{VICENTE FERRER: VIDA Y TRATADOS LÓGICOS}

Dentro del complejo contexto histórico del siglo XIV la figura de Vicente Ferrer (13501419) ocupa un lugar de particular relieve. Son bien conocidas sus extraordinarias dotes de taumaturgo y predicador infatigable (que le valió el calificativo de «apóstol de Europa»); hábil mediador político como lo demuestra su intervención en el compromiso de Caspe; confesor del papa Pedro de Luna en la corte de Avignon hasta que le retiró su apoyo. ${ }^{8} \mathrm{Sin}$ embargo, la figura de Ferrer como lógico y filósofo sólo recientemente ha sido redescubierta. En efecto, hasta principios de este siglo se consideraban perdidas las dos únicas obras filosóficas que escribió. Hoy contamos tan sólo con tres manuscritos del Tractatus de

7 Cfr. Gilson, E., La filosofía en la Edad Media, p. 657.

8 Para un completo análisis histórico y biográfico de S. Vicente Ferrer, cfr. Garganta, J.M., y Forcada, V., Biografía y escritos de San Vicente Ferrer, BAC, Madrid, 1956; Gorce, M.M., Saint Vincent Ferrier (13501419), Libraire Plon, Paris 1924; Idem. s.v. «Vincent Ferrier», en DTC, Paris 1950, t. XV, cols. 3033-3045. Les bases de l'étude historique de Saint Vincent Ferrier, Paris, 1933. 
Suppositionibus terminorum (en Madrid, Viena y Pavía) ${ }^{9}$ y uno (en Viena) ${ }^{10}$ de la Quaestio Solemnis de Unitate Universalis.

Vicente Ferrer ingresa en la Orden de Predicadores de Valencia en 1367, y al año siguiente el Capítulo Provincial celebrado en Tarragona le asigna al Estudio General de Barcelona como estudiante de Lógica. Terminado el curso de 1368-1369, se traslada a Lérida como estudiante del Studium naturarum. El curso 1370-1371 está en Lérida ocupándose de la enseñanza de Lógica como magister logicorum, puesto que ocupó hasta el curso siguiente, en el que se traslada a Barcelona para estudiar Teología y Sagrada Escritura. ${ }^{11}$ En 1376 se dirige a Toulouse para especializarse en Sagrada Teología, y regresará pocos meses después a Valencia.

Las dos obras filosóficas del dominico valenciano se sitúan entre los años 1371 y 1375 , es decir, cuando nuestro autor contaba 21 o 25 años; probablemente las escribiera en Lérida durante su estancia como profesor de Lógica. ${ }^{12}$ La Universidad de Lérida (fundada en 1300) pasa por ser un notable foco nominalista por esas fechas; Ferrer enseñaría allí apenas veinticinco años después de la muerte de Ockham, y ya su doctrina había arraigado con fuerza en gran número de universidades europeas.

No ha dejado de llamar la atención de los estudiosos de la obra vicentina, la temprana edad de composición de sus tratados filosóficos, sobre todo si tenemos en cuenta la originalidad y madurez de su pensamiento manifestadas en estas obras de juventud. De hecho, Brettle llega a poner en duda la autoría de estos tratados basándose para ello en la desproporción que se observa entre el contenido de las obras y la corta edad del dominico valenciano. ${ }^{13}$ Pero la cuestión de la autenticidad de los tratados del maestro Ferrer parece quedar definitivamente zanjada con los estudios posteriores de Lechat y Gorce, ${ }^{14}$ que aducen en favor de la paternidad de S. Vicente de estos tratado, el hecho de que ya en la biografía de su proceso de beatificación realizada por Ranzano en 1455 se reconoce explícitamente la autoría de los mismos.

9 Madrid, Biblioteca Nacional, cod. 3368. Cfr, Beltrán de Heredia, V., «Los manuscritos de Santo Tomás de la Biblioteca Nacional de Madrid», en La Ciencia Tomista, 34 (1926), p. 97; Viena, Dominikanerkonvent, 49/271; Pavía, Biblioteca Universitaria, 365.

10 Viena, Dominikanerkonvent, 49/271.

11 Para una exposición detallada de la fundación e historia de estas primeras universidades españolas cfr. Rashdall, H; Ponicke, F.M. y Emden, A.B. (edit.), The Universities of Europe in the Middle Ages (vol. II), new edition, Oxford University Press, 1969, pp. 90-109.

12 Sobre la discusión del lugar y fecha de composición de estos tratados, cfr. García Miralles, M., «Escritos filosóficos de San Vicente Ferrer», en Estudios Filosóficos, 4 (1955), pp. 279-284; Forcada, V., "Momento histórico del tratado De Suppositione de San Vicente Ferrer», en Escritos del Vedat, 3 (1973), pp. 60-65; y más recientemente la introducción de A. Robles a los Tratados Filosóficos de San Vicente Ferrer, pp. 5-17.

13 Cfr. Brettle, P., San Vicente Ferrer, und sein litterarische Nachlass, in West. Aschendorf, Münster 1924, p. 33. La razón decisiva de su argumentación es el hecho de que, según él, estas obras no comienzan a ser atribuidas a S. Vicente hasta 1484.

14 Cfr. Lechat, P., «Bulletin des publications hagiographiques», en Anallecta Bollandiana, 44 (1926), pp. 217-218; Gorce, M.M., Les bases de l'étude historique de Saint Vincent Ferrier, p. 2; Carreras Artau, J. y T., Historia de la Filosofía Española (II). Filosofía cristiana de los siglos XIII al XV, Asociación Española para el Progreso de las Ciencias, Madrid, 1943, pp. 453-456. 
La historia de los tratados filosóficos de Ferrer cuenta con una intrincada evolución. La biografía de Ranzano dedica grandes elogios al De Suppositionibus. ${ }^{15}$ Por otro lado, biógrafos del santo (Teixidor y Baltasar Sorió) y los catálogos de escritores del reino de Valencia de estos años mencionan estas obras juveniles de Vicente Ferrer. Esto quiere decir que los tratados ferrerianos fueron conocidos y usados en mayor o menor medida a lo largo de los siglos $\mathrm{XV}$ y XVI. Nos han llegado los testimonios de algunos lógicos de esa época en los cuales se cita expresamente alguna de las obras filosóficas de Ferrer. En concreto, Ángel Estañol en su Opera Logicalia (1504) y Pedro Nigro en el Clypeus thomisticarum (1504) lo mencionan expresamente; del mismo modo Mengo B. Faventino en su Comentario a las Súmulas de Pablo de Venecia (1520) y más adelante Juan Sánchez Sedeño en la Lógica $(1600) .{ }^{16}$

Sin embargo, a partir de los primeros años del siglo XVII, las obras lógicas de Vicente Ferrer parecen haber caído en el olvido, y se consideran completamente perdidas, ${ }^{17}$ así como las anotaciones que nuestro autor hizo a algunas cuestiones de la Suma Teológica de Santo Tomás. ${ }^{18} \mathrm{Y}$ también en el siglo XIX se daban por perdidas, según testimonia un biógrafo del santo. ${ }^{19}$

Hemos de esperar a 1909, cuando el P. Fages hace volver a la luz los manuscritos que se consideraban perdidos. La edición contiene numerosos errores y maneja tan sólo el manuscrito de Viena, pero hace posible el redescubrimiento de la doctrina contenida en estas obras ferrerianas. Con todo, hasta algunos años más tarde no encontramos una exposición detallada de los opúsculos de Vicente Ferrer; se la debemos al P. Gorce, que dedica gran atención a estas obras en la voz «Realisme» del Dictionnaire de Théologie Catholique..$^{20}$ Por otro lado, los filósofos neoescolásticos, a pesar de la afinidad con la doctrina tomista, no lo citarán de modo expreso. Sólo Maritain le dedica una escueta mención en su manual de lógica. ${ }^{21}$

15 Particularmente significativas resultan estas palabras: «Inter studiorum vero eius tempora, cum esset 24 annorum, edidit insigne opus De dialecticis suppositionibus: in quo quisque manifeste videre potest quanta fuerit aucthoris peritia si consideretur in ipso opusculo multa esse praeclarissima, non solum ex media philosophia verum etiam ex profundissima Theologia delecta». Citado por V. Forcada, «Momento histórico del tratado...», p. 64. 56.

16 Cfr. Muñoz Delgado, V., Lógica hispano-portuguesa hasta 1600, RHCEE, Salamanca 1972, pp. 55-

17 Así por ejemplo lo manifiesta Vidal y Micó: «[...] compuso un trabajo ingenioso y erudito, De las suposiciones dialécticas, y otro De la naturaleza del universal, en que manifiesta bien la sutileza y fondos de su ingenio, tanto en punto de filosofía como de Sagrada Teología. Alaban mucho estas obras los antiguos que las vieron, como Ranzano y Flaminio. Ahora no se hallan. Debieron perderse, como otras de ese género, partos de varones excelentes, en la borrasca que en España padecieron los tratados de Súmulas en tiempo del Maestro Soto». Citado por V. Forcada, «Momento histórico del tratado De Suppositione...», p. 65.

18 Estas anotaciones se han publicado modernamente gracias al trabajo de García Miralles; Cfr. García Miralles, M., «San Vicente Ferrer, anotador de Santo Tomás», en Revista Española de Teología, 15 (1955), pp. 88-101.

19 Crr. Bayle, A., Vie de Saint Vincent Ferrier, Paris, 1855, p. 327.

20 Cfr. Gorce, M.M., s.v. «Realisme. Vincent Ferrier», en DTC, Paris, 1937, t. XIII-2. ${ }^{\text {a }}$ parte, cols. 18641869.

21 Maritain, J., El orden de los conceptos, Club de Lectores, Buenos Aires, 1956, p. 92. 
En 1943 aparece una sucinta exposición de las obras ferrerianas en la Historia de la Filosofia de Carreras Artau, ${ }^{22}$ y en 1952 Ivo Thomas realiza un estudio detallado del Tractatus desde el punto de vista de la lógica formal. ${ }^{23} \mathrm{~A}$ partir de entonces, y con un interés creciente por parte de estudiosos de la lógica y semiótica medievales, se incluye de modo habitual en los trabajos de historia de la lógica. De las obras ferrerianas contamos con la edición citada de Fages publicada en 1909. Como mencioné anteriormente, Trentman ha llevado a cabo una edición crítica del Tractatus de Suppositionibus elaborada con los manuscritos de Madrid, Pavía y Viena ${ }^{24}$ y de la Quaestio De Unitate Universalis. Por último, la reciente traducción castellana antes citada, corrige en algunos fragmentos las ediciones críticas de Trentman.

\section{FUENTES Y OBJETO DE ESTUDIO}

En la primera obra lógica del dominico valenciano - la Quaestio De Unitate Universalis- se ocupa del problema de los universales, planteado desde una perspectiva realista moderada. Se trata - según parece- de un ejercicio escolar de Ferrer y posee la estructura característica de una quaestio medieval. Se divide en cuatro apartados: $\left.{ }^{25} 1 . .^{\text {g) }}\right)$ Presentación del problema que se quiere resolver: si la unidad del universal es real o no. Se incluyen doce argumentos realistas exagerados según los cuales la unidad del universal es real; 2.) Los sed contra: en los cuales S. Vicente recoge catorce argumentos (la mayor parte de ellos tomados de los nominalistas) en contra de la unidad real del universal; 3..9) Solución propuesta por el autor: la unidad del universal no es real sino de razón, aunque con fundamento in re; $\left.4 .^{\circ}\right)$ Respuesta a las argumentaciones realistas exageradas en favor de la unidad real del universal.

En cuanto a las fuentes de la Quaestio se han de distinguir las fuentes explícitas y las implícitas. El autor más citado expresamente es Aristóteles: 24 citas en total ${ }^{26}$ Trentman ha hecho notar la ausencia de referencias explícitas a Santo Tomás, pero hemos de reconocer con él que la doctrina ferreriana es deudora por completo de la del Doctor Angélico. ${ }^{27}$

Más interesante y significativo resulta el análisis textual del Tractatus de Suppositionibus. Ferrer se propone realizar un estudio acerca de la noción y clasificación de la suppositio;

22 Cfr. Carreras Artau, J. y T., Historia de la Filosofía Española, pp. 453-456.

23 Cfr. Thomas, I., «Saint Vincent Ferrer's De Suppositionibus», en Dominican Studies, 5 (1952), pp. 88101.

24 Sobre esta edición crítica, vid. la reseña publicada por Bos, E., en Vivarium, 18 (1980), pp. 79-80.

25 Para una exposición del contenido de esta obra cf. Beuchot, M., «El problema ontológico de los universales en San Vicente Ferrer (1350-1419)», en Escritos del Vedat, 20 (1990), pp. 375-384.

26 Las citas se distribuyen del siguiente modo: Analíticos Posteriores [5], Metafísica [5], Física [5], Tópicos [3], Analíticos Primeros, Refutaciones Sofísticas, Categorías, Ética a Nicómaco, De coelo et mundo, y De generatione et corruptione [1]. Aparte del Estagirita cita en una ocasión a Boecio, Avicena y el Liber de Causis.

27 Cfr. Trentman, J.A., «The Questio De Unitate Universalis of Vincent Ferrer», p. 115. 
para ello distribuirá su estudio en diez capítulos precedidos por un proemio. En éste presenta el contenido del tratado relacionándolo con la cuestión de los universales y el realismo moderado de Santo Tomás; en los capítulos que siguen examina la noción de suppositio y los diversos tipos de suposiciones que propone.

En cuanto a las fuentes del Tractatus distinguiremos en esta ocasión entre las fuentes estrictamente lógicas (no citadas expresamente) y aquellas de carácter filosófico citadas de modo explícito. Entre las primeras debemos mencionar en primer lugar las obras de Guillermo de Shyreswood y Walter Burleigh. Shyreswood es una fuente indirecta pero de gran relevancia a la hora de establecer la noción general y la división de las suposiciones, aunque Ferrer corrige en algunos aspectos la propuesta de Shyreswood. El influjo de Burleigh nos parece más patente: la definición de S. Vicente de la suposición está tomada del Puritate de Artis Logicae, si bien es cierto que la reformula de modo original. Por otro lado, multitud de ejemplos y tratamientos ferrerianos están tomados de la lógica de Burleigh, muchos de ellos recogidos de la disputa con Ockham. No obstante, se alejará explícitamente de las posturas extremas de Burleigh acerca del problema de los universales. Por otra parte, se destaca también el influjo del Tractatus de Pedro Hispano, y quizás de él tomara la noción de suposición natural aunque planteada desde una perspectiva del todo nueva. ${ }^{28}$

Por lo que se refiere a las fuentes filósoficas es manifiesta la filiación tomista de nuestro autor. Así, en el prólogo del Tractatus afirmará refiriéndose a la solución del Doctor Angélico al problema de los universales: «[...] no merece llamarse opinión, sino sentencia y verdad, porque es ilustrada con la ciencia de la verdad, aprobada con la sentencia de la autoridad, corroborada por el sentido del equilibrio [...]. Ésta es la sentencia del glorioso Doctor Santo Tomás, quien dice que el universal materialmente es una cosa, y formalmente es una intención». ${ }^{29}$ Y poco más adelante afirmará que su propósito no es otro que el de llevar a cabo una teoría de la suposición de acuerdo con los principios filosóficos tomistas, frente a la teorías de Burleigh y Ockham: «[...] porque Walter Burleigh trató de las suposiciones según la primera opinión extrema del universal; $y$, porque trató también según la otra opinión extrema el hermano Guillermo de Ockham y sus seguidores, mucho más se habrá de decir del universal y tratar de las suposiciones, según la sentencia verdadera y media. [...] sólo me propongo tratar de las suposiciones según la sentencia del mencionado Santo Doctor, con el propósito de utilizar sus mismas palabras, en cuanto me sea posible, y así proceder a tratar de las suposiciones de los términos, según esta sentencia verdadera». ${ }^{30}$

28 Muñoz Delgado piensa que la lógica de $\mathrm{S}$. Vicente es el fruto de una actitud ecléctica entre la lógica de Pedro Hispano y Walter Burleigh, y en cierto sentido también de Ockham y Guillermo de Shyreswood. Cfr. Muñoz Delgado, V., Lógica hispano-portuguesa hasta 1600, p. 55.

29 Tractatus de Suppositionibus (TS), pp. 43-45 (87-88). Cito por la traducción castellana, añadiendo entre paréntesis la paginación de Trentman.

30 TS, p. 45 (88). Trentman hace hincapié en el exceso de «humildad intelectual» mostrado por $S$. Vicente. Ciertamente seguirá con fidelidad la doctrina del Aquinate, pero introduce enfoques y tratamientos originales que no se encuentran así formulados en Santo Tomás. Cfr. TS, ed. Trentman, «Introduction II», pp. $17-18$. 
Junto a la autoridad de Santo Tomás y Aristóteles cita a Alberto Magno, Boecio, Avicena, Averroes y al maestro Herveo. ${ }^{31}$ Por otro lado cabría señalar algunos autores de la época pertenecientes a la Corona de Aragón - dominicos en su mayoría- que pudieron haber ejercido alguna influencia en la redacción de los tratados ferrerianos; tal es el caso de Pedro de Aragón, Juan Fort, Nicolás Eymerich, Juan Monzón, Pedro Soplana, Antonio Ginebreda, Antonio Canals, Pedro Tomás o Guillermo Rubió. ${ }^{32}$

Un pormenorizado análisis de las fuentes mencionadas a lo largo del tratado viene a confirmar la filiación aristotélico-tomista del dominico valenciano. Aparte de otras citas no explícitas, encontramos 74 citas de Aristóteles ${ }^{33}$ y 39 citas de obras de Santo Tomás. ${ }^{34} \mathrm{El}$ -elenco de citas se completa con Averroes [5] y con otros autores a los que tan sólo cita una vez: Boecio, Porfirio, Pedro Elías, Alfarabí, Egidio Romano y el Liber de Causis. Como se puede fácilmente concluir, la formación filosófica de Ferrer está claramente mediatizada por el pensamiento de Tomás de Aquino, y a través suyo recibirá toda la tradición clásica (aristotélica sobre todo) y medieval.

\section{ORIGINALIDAD DEL PLANTEAMIENTO FERRERIANO E INFLUJO POSTERIOR}

A pesar del peso explícito que recae sobre Ferrer del pensamiento del Aquinatense, lo cierto es que presenta una notable novedad respecto a su maestro, fundamentalmente por dos hechos. En primer lugar, porque Tomás de Aquino no elaboró un pensamiento sistemático sobre lógica o semiótica acorde con su metafísica realista, sino que se limitó principalmente a emplear la lógica comúnmente aceptada por sus contemporáneos y que aprendió en París, seguramente de la mano de Gulllermo de Shyreswood. Esta tarea sistematizadora fue la llevada a cabo por el maestro Ferrer; teniendo presente la metafísica tomista se propone elaborar una teoría de la suposición coherente con su ontología. Y es que, en segundo término, el desarrollo de una semántica realista se hacía particularmente urgente

31 Herveo Natalis - Doctor rarus- (?-1323). Fue maestro regente de Saint Jacques en 1307. Defendió la doctrina tomista contra Enrique de Gante, Jacobo de Metz, Escoto, Durando de San Porciano y Pedro Aureolo. En 1318 fue elegido maestro general de la orden, trabajando intensamente para la canonización de Santo Tomás. Criticó duramente a los dominicos que abandonaban la doctrina tomista y escribió una obra Defensa doctrinae D. Thomae, que constituye la primera apología de la Suma Teológica, defendiendo el valor científico de la Teología: en ella se ocupó del valor real de los universales. Cfr. Fraile, G., Historia de la Filosofia, p. 483.

32 Cfr. las referencias de estos autores contenidas en Muñoz Delgado, V., Lógica hispano-portuguesa hasta 1600; Robles, L., Escritores Dominicos de la Corona de Aragón (s. XIII-XV), Salamanca, 1972.

33 Estas citas se distribuyen del siguiente modo: Analíticos Posteriores [20]; Tópicos [13]; De Anima [10]; Física [8]; Primeros Analiticos [6]; De Interpretatione [5]; De Generatione [5]; Categorías [3] y otras obras [4].

34 Suma Teológica [7]; In Peri Hermeneias [7]; In De Anima [4]; Super Librum de Causis [4]; In Librum Sententiarum [4]; De Fallaciis [4]; De Ente et Essentia [3]; otras obras [6]. 
después de que el conceptualismo ockhamiano había conseguido sistematizar de modo coherente una teoría de la significación y de la suposición (que es casi tanto como decir toda una filosofía del lenguaje) centrada en una metafísica del singular, eliminando de la consideración ontológica el problema de la naturaleza. Por eso, Ferrer no sólo es un sistematizador de la semántica tomista, sino, sobre todo, un autor que se sitúa en abierto diálogo frente a toda una corriente de pensamiento «moderna» que tendrá como piedra de toque una filosofía del lenguaje nominalista.

Desde esta perspectiva, los enfoques novedosos de Vicente Ferrer afectan tanto a su noción de la significatio y suppositio (que son una respuesta a toda la tradición lógica medieval desde Pedro Hispano a Guillermo de Ockham), como a la estructura proposicional (frente a la teoría de los «dos nombres» propuesta por los nominalistas), pasando por la clasificación de la suppositio y su criterio clasificatorio, la interpretación lingüística de la suposición material que le permite la distinción entre mención y uso de un término ${ }^{35} \mathrm{y}$ sobre todo la suposición natural, verdadero eje de toda su semántica realista, cuya originalidad e importancia no ha pasado inadvertida a los historiadores de la lógica medieval. ${ }^{36} \mathrm{La}$ explicación detallada de cada uno de estos aspectos nos llevaría muy lejos; pero todos ellos manifiestan un declarado interés de establecer una filosofía del lenguaje de corte realista frente al nominalismo conceptualista.

Sin embargo, la incidencia de la propuesta ferreriana fue mínima en la escolástica «tomista» posterior, como es fácil advertir en algunos autores paradigmáticos como son por ejemplo Domingo de Soto y Juan de Santo Tomás, que intentaron construir una lógica desde los presupuestos tomistas. Ya una primera comparación de las definiciones de Domingo de Soto (1494-1560) y Vicente Ferrer, nos pone frente a dos concepciones bastante divergentes sobre la naturaleza y clasificación de la suposición. Contrariamente al maestro Soto, el dominico valenciano propone una definición «sintáctica» de la suppositio (basada en la relación entre el sujeto y el predicado), el carácter activo de la noción de suppositio y la negación de proposiciones con sujeto no suponente. ${ }^{37}$ Por su parte Juan de Santo Tomás (1589-1644), que junto con la tradición escolástica hispánica del XVI rechaza decididamente el nominalismo medieval apoyándose explícitamente en la filosofía tomista, presenta

35 Cfr. Bochenski, I.M., Historia de la lógica formal, Gredos, Madrid, 1966; López García, A., «Nota para la historia de la lingüística española: la contribución de San Vicente Ferrer» en Philologica, II (1989), pp. 389-398.

36 Las referencias bibliográficas son abundantes sobre este tema; cfr. Barth, E.M., The logic of the Articles in Traditional Philosophy. A Contribution to the Study of Conceptual Structures, Dordrecht, 1974; De Rijk, L.M., La Philosophie au Moyen Âge, Leiden-E.J. Brill, 1985; De Rijk,. L.M., «The Development of Suppositio Naturalis in Medieval Logic (I)», en Vivarium, 9 (1971), pp. 71-107; De Rijk,, L.M., "The Development of Suppositio Naturalis in Medieval Logic (II)", en Vivarium, 11 (1973), pp. 43-79; Ducrot, O., "Quelques implications linguistiques de la théorie médiévale de la supposition», en History of linguistic Thought and Contemporary linguistics, Walter de Gruyter, Berlin, 1976, pp. 189-227; Kneale, W. y M., El desarrollo de la lógica, Tecnos, Madrid, 1980; Pinborg, J., Logica e Semantica nel Medioevo, Boringheri, Torino, 1984.

37 Una exposición más detallada sobre la teoría de la suposición de Ferrer en comparación con la propuesta de Domingo de Soto y Juan de Santo Tomás puede encontrarse en García Cuadrado, J.A., Hacia una semántica realista. La filosofía del lenguaje de S. Vicente Ferrer, Eunsa, Pamplona, 1994 (cap. VII). 
diferencias en absoluto epidérmicas con el planteamiento ferreriano: la suposición del sujeto y del predicado (frente a la suposición exclusiva del sujeto según Ferrer), la inclusión de la suposición natural dentro de la suposición personal, y no un tipo específicamente diverso de éste, son sólo algunas de las explícitas divergencias de una y otra propuesta. ${ }^{38}$

Curiosamente, la lógica de Ferrer tuvo más eco entre los escolásticos «no tomistas» posteriores, algunos de los cuales retomaron nociones ferrerianas de su teoría de la suposición. Por ejemplo, la original división introducida por S.Vicente entre suposición discreta y común aplicada también a la suposición material, se encuentra recogida por Pablo Véneto, y como testimonia su comentador Mengo B. Faventino, la pudo haber retomado del maestro Ferrer.$^{39}$ También parece encontrarse esta distinción en otros autores de la tradición lógica italiana, como son Pablo de Venecia y Pablo de Pérgula. ${ }^{40}$ Por otro lado, en esta misma tradición lógica italiana, concretamente en Pedro de Mantua, se recoge el carácter activo de la significatio, tal como había propuesto Ferrer al comienzo de su tratado. ${ }^{41}$

Sintetizando, se puede decir que han sido dos las aportaciones principales de la obra ferreriana a la lógica posterior: el valor atemporal de la suposición natural, y la distinción, dentro de la suposición material, en común y discreta. La primera de estas aportaciones ha sido puesta en conexión con la doctrina fregeana del «tercer reino: objetivo y no real» viendo el paralelismo con las proposiciones con suppositio naturalis, que son el objeto propio del saber científico según el lógico valenciano. ${ }^{42}$ Por su parte, la distinción de la -suposición material en común y discreta es repropuesta por Peirce con la distinción entre type-sign y token-sign ${ }^{43}$

Sin embargo, la propuesta de Ferrer, deudora sólo en parte de la de Shyreswood y Burleigh, se muestra original, y al mismo tiempo aislada e ignorada en la lógica tomista neoescolástica. ${ }^{44}$ Cabe preguntarse, a la luz de lo expuesto, hasta qué punto los lógicos

38 Cfr. Trentman, J.A., «Scholasticism in the Seventeenth Century», en The Cambridge History..., pp. 818-819.

39 Cfr. Trentman, J.A., «Introduction» I, p. 12; Ashworth, E.J., «The Doctrine of Supposition in Sixteenth and Seventeenth...», p. 260.

40 Cfr. Karger, E., «La supposition materialle comme supposition significative: Paul de Venise, Paul de Pergula», en English Logic in Italy in the 14th. and 15th. Centuries. Acts of the 5th. European Symposium on Medieval Logic and Semantics, Bibliopolis, Napoli, 1982, pp. 331-341.

41 Cfr. Bos, E.P. «Peter of Mantua's tract on appellatio and his interpretation of inmanent forms», en English Logic in Italy..., p. 244.

42 Otras coincidencias entre los planteamientos de Frege y Ferrer han sido puestas de manifiesto por Beuchot, M. «Un antecesor de Frege: Vicente Ferrer (s. XIV) y la estructura proposicional», pp. 389-397; Idem, «Un caso de influencia de la estructura ontológica entitativa sobre la estructura lógica proposicional: San Vicente Ferrer (s. XIV), antecesor de Frege», en Humanidades, 9 (1986), pp. 55-66; Jiménez Cataño, R., Semántica y Racionalidad en Frege. Un estudio desde las operaciones mentales, Minos, México, 1991, pp. 235-249.

43 Cfr. López García, A., «Nota para la historia de la lingüística española: la contribución de San Vicente Ferrer» en Philologica, II (1989), pp. 389-398.

44 «Il est remarquable que beacoup de manuels de scolastique, bien qu'ils soient d'inspiration thomiste, donnent pour définition générale de la supposition celle de Pierre d'Espagne ou d'Occam, alors que cette définition était, au Moyen-Âge même, explicitament contestée par les thomistes, par exemple par Saint Vincent Ferrier». Ducrot, O., «Quelques implications linguistiques...», p. 191, n. 5. 
neoescolásticos «tomistas» han sido fieles a la teoría predicativa propuesta por el Aquinate, sobre todo en lo referente a la «teoría de los dos nombres» resultante de conceder suposición tanto al sujeto como al predicado de la proposición. Además, el núcleo de su doctrina acerca de los tipos de suposición se ve notablemente deformada en la escolástica tomista posterior: se pierde de vista una jerarquía clara de los tipos de suposición, se oscurece el papel de la suposición natural como analogado principal y se difumina la distinción con la suposición personal.

En la lectura de los textos ferrerianos se advierte cómo todas estas cuestiones «lógicas» poseen un trasfondo gnoseológico que no puede ser pasado por alto. La lógica neoescolástica, quizás influenciada por el indudable avance que supusieron los tratamientos lógicos nominalistas, acepta e incorpora nociones provenientes de esa tradición, sin someterlos a crítica intentándolos conciliar con una gnoseología tomista. Pero creo que este intento desfigura el sentido de la semántica realista implícitamente presente en Tomás de Aquino. No debemos olvidar que tanto la lógica como la filosofía del lenguaje necesitan de una explicación y fundamentación que se halla más allá de sus fronteras: aquellas que limitan con la ontología y con la teoría del conocimiento.

\section{BIB̀LIOGRAFÍA SOBRE LA OBRA FILOSÓFICA DE S. VICENTE FERRER}

A. Ediciones de los tratados filosóficos de San Vicente Ferrer:

FAGES, H.D., Oeuvres de Saint Vincent Ferrier, vol. I., Paris, 1909.

FORCADA, V., Tratados filosóficos de San Vicente Ferrer; traducción castellana V. Forcada; introducción y notas A. Robles. Provincia Dominicana de Aragón, Valencia, 1987.

TRENTMAN, J.A., «The Questio de Unitate Universalis of Vincent Ferrer», en Medieval Studies, 44 (1982), pp. 122-137.

TRENTMAN, J.A., Tractatus de Suppositionibus, Grammatica Speculativa, Sprachtheorie und Logik des Mittelalters 2: Stuttgart-Bad Cannstatt, 1977.

B. Monografías sobre su obra filosófica o con referencias a la misma

BARTH, E.M., The Logic of the Articles in Traditional Philosophy. A Contribution to the Study of Conceptual Structures, Dordrecht, 1974.

BAYLE, A., Vie de Saint Vincent Ferrier, Paris, 1855.

BEUCHOT, M., La filosofia del lenguaje en la Edad Media, UNAM, México, 1984.

BEUCHOT, M., Lógica y Ontología, Depto. Editorial Universidad de Guadalajara, Guadalajara, 1986.

BEUCHOT, M., Aspectos históricos de la semiótica y ta filosofía del lenguaje, UNAM, México 1987.

BOCHENSKI, I.M., Historia de la lógica formal, Gredos, Madrid, 1966.

BRETTLE, P., San Vicente Ferrer, und sein litterarische Nachlass, in West., Aschendorf, Münster, 1924.

CARRERAS ARTAU, J. y T., Historia de la Filosofía Española: Filosofia Cristiana de los siglos XIII al XV, Asociación Española para el Progreso de las Ciencias, Madrid, 1939-1943. 
DE RIJK, L.M., La Philosophie au Moyen Âge, Leiden-E.J. Brill, 1985.

FRAILE, G., Historia de la Filosofía Española. Desde de la época romana hasta fines del siglo XVII, BAC, Madrid, 1971.

GARGANTA, J.M., y FORCADA, V., Biografía y escritos de San Vicente Ferrer, BAC, Madrid, 1956.

GORCE, M.M., Saint Vincent Ferrier (1350-1419), Libraire Plon, Paris, 1924.

GORCE, M.M., Les bases de l'étude historique de Saint Vincent Ferrier, Paris, 1933.

JIMÉNEZ CATAÑO, R., Semántica y Racionalidad en Frege. Un estudio desde las operaciones mentales, Minos, México, 1991, pp. 235-249.

KNEALE, W. y M., El desarrollo de la lógica, Tecnos, Madrid, 1980.

MARITAIN, J., El orden de los conceptos, Club de Lectores, Buenos Aires, 1967.

PINBORG, J., Logica e Semantica nel Medioevo, Boringheri, Torino, 1984.

C. Artículos

BEUCHOT, M., «Un antecesor de Frege. Vicente Ferrer (s. XIV) y la estructura proposicional», en Escritos del Vedat, 16 (1986), pp. 389-397.

BEUCHOT, M., «El Problema ontológico de los universales en San Vicente Ferrer (1350-1419)» en Escritos del Vedat, 20 (1990), pp. 375-384.

BEUCHOT, M., «Un caso de influencia de la estructura ontológica entitativa sobre la estructura lógica proposicional: San Vicente Ferrer (s. XIV), antecesor de Frege», en Humanidades, 9 (1986), pp. 55-66.

BOS, E., «Tractatus de Suppositionibuss; ed. crítica de J.A. Trentman», en Vivarium, 18 (1980), pp. 79-80.

DE RIJK, L.M., «The Development of Suppositio Naturalis in Medieval Logic (I)», en Vivarium, 9 (1971), pp. 71-107.

DE RIJK, L.M., «The Development of Suppositio Naturalis in Medieval Logic (II)», en Vivarium, 11 (1973), pp. 43-79.

DUCROT, 0., «Quelques implications linguistiques de la théorie médiévale de la supposition», en History of linguistic Thought and Contemporany linguistics, Walter de Gruyter, Berlin, 1976, pp. 189-227.

FORCADA, V., «Momento histórico del tratado De Suppositione de San Vicente Ferrer», en Escritos del Vedat, 3 (1973), pp. 37-89.

GARCÍA MIRALLES, M., «Escritos filosóficos de San Vicente Ferrer», en Estudios Filosóficos, 4 (1955), pp. 279-284.

GARCÍA MIRALLES, M., «San Vicente Ferrer, anotador de Santo Tomás», en Revista Española de Teología, 15 (1955), pp. 88-101.

GORCE, M.M., «Realisme. Vincent Ferrer», en DTC, Paris, 1937, t. XIII-2." parte, cols. 1864-1869.

GORCE, M.M., «Vincent Ferrier (saint)», en DTC, Paris, 1950, t. XV, cols. 3033-3045.

LECHAT, P., «Bulletin des publications hagiographiques», en Anallecta Bollandiana, 44 (1926), pp. 138-240.

LÓPEZ GARCÍA, A., «Nota para la historia de la lingüística española: la contribución de san Vicente Ferrer», en Philologica, II (1989), pp. 389-398.

POVEDA, E., «El tratado De Suppositionibus dialecticis de S. Vicente Ferrer y su significación histórica en la cuestión de los universales», en Anales del Seminario de Valencia, 6 (1963), pp. 5-88. 
SCOTT, T.K., «John Buridan on the Objects of Demonstrative Science», en Speculum, 40 (1965), pp. 654-673.

THOMAS, I., «Saint Vincent Ferrer's De Suppositionibus», en Dominican Studies, 5 (1952), pp. 88101.

TRENTMAN, J.A., «Vincent Ferrer on the Logician as Artifex Intellectualis», en Franciscan Studies, 25 (1965), pp. 322-337.

TRENTMAN, J.A., «Lesniewski's Ontology and Some Medieval logicians», en Notre Dame of Formal Logic, 7 (1966), pp. 361-364.

TRENTMAN, J.A., «Predication and Universals in Vincent Ferrer's Logic», en Franciscan Studies, 28 (1968), pp. 47-62.

TRENTMAN, J.A., «Vincent Ferrer and His Fourteenth-Century Predecessors on a Problem of Intentionality», en Actes du Quatrième Congrès International de Philosophie Médiévale, Montreal, 1969, pp. 949-956.

TRENTMAN, J.A., «Ockham on Mental», en Mind, 79 (1970), pp. 586-590.

TRENTMAN, J.A., «The idea of signification in Vincent Ferrer's Logic», en Actas del V Congreso Internacional de Filosofía Medieval, Madrid, 1972, pp. 1301-1310.

TRENTMAN, J.A., «Speculative Grammar and Transformational Grammar: A Comparation of Philosophical Pressuppotions» en History of Linguistic Thought and Contemporary Linguistics, Walter de Gruyter, Berlin, 1976, pp. 279-301.

TRENTMAN, J.A., «On Interpretation, Lesniewski's Ontology, and the study of Medieval Logic»; en Journal of the History of Philosophy, 14 (1976), pp. 217-222.

TRENTMAN, J.A., «Scholasticism in the Seventeenth Century», en The Cambridge History of Later Medieval Philosophy, Cambridge University Press, 1982, pp. 818-837.

TRENTMAN, J.A., «The Questio de Unitate Universalis of Vincent Ferrer», en Medieval Studies, 44 (1982), pp. 110-121.

TRENTMAN, J.A., «Ferrer», en Theologische Realenzyklopädie, ed. H. R. Balz, t. XI, Walter de Gruyter, Berlin, 1983, pp. 91-93.

VALDIVIA, B., «La suposición semántica en Vicente Ferrer», en Analogía, 1, n.. 2 (1987), pp. 85-91. 